\title{
EXTENSION AND GENERALIZATION INEQUALITIES INVOLVING THE KHATRI-RAO PRODUCT OF SEVERAL POSITIVE MATRICES
}

\author{
ZEYAD ABDEL AZIZ AL ZHOUR AND ADEM KILICMAN
}

Received 15 February 2005; Accepted 16 October 2005

Recently, there have been many authors, who established a number of inequalities involving Khatri-Rao and Hadamard products of two positive matrices. In this paper, the results are established in the following three ways. First, we find generalization of the inequalities involving Khatri-Rao product using results given by Liu (1999), Mond and Pečarić (1997), Cao et al. (2002), Chollet (1997), and Visick (2000). Second, we recover and develop some results of Visick. Third, the results are extended to the case of KhatriRao product of any finite number of matrices. These results lead to inequalities involving Hadamard product, as a special case.

Copyright (c) 2006 Z. A. Al Zhour and A. Kilicman. This is an open access article distributed under the Creative Commons Attribution License, which permits unrestricted use, distribution, and reproduction in any medium, provided the original work is properly cited.

\section{Introduction}

Consider matrices $A$ and $B$ of order $m \times n$ and $p \times q$, respectively. Let $A=\left[A_{i j}\right]$ be partitioned with $A_{i j}$ of order $m_{i} \times n_{j}$ as the $(i, j)$ th block submatrix and let $B=\left[B_{k l}\right]$ be partitioned with $B_{k l}$ of order $p_{k} \times q_{l}$ as the $(k, l)$ th block submatrix $\left(m=\sum_{i=1}^{t} m_{i}, n=\right.$ $\left.\sum_{j=1}^{d} n_{j}, p=\sum_{k=1}^{u} p_{k}, q=\sum_{l=1}^{v} q_{l}\right)$. For simplicity, we say that $A$ and $B$ are compatible partitioned if $A=\left[A_{i j}\right]_{i, j=1}^{t}$ and $B=\left[B_{i j}\right]_{i, j=1}^{t}$ are square matrices of order $m \times m$ and partitioned, respectively, with $A_{i j}$ and $B_{i j}$ of order $m_{i} \times m_{j}\left(m=\sum_{i=1}^{t} m_{i}=\sum_{j=1}^{t} m_{j}\right)$.

Let $A \otimes B, A \circ B, A \Theta B$, and $A * B$ be the Kronecker, Hadamard, Tracy-Singh, and Khatri-Rao products, respectively, of $A$ and $B$. The definitions of the mentioned four matrix products are given by Liu in $[5,6]$ as follows:

(i) Kronecker product

$$
A \otimes B=\left[a_{i j} B\right]_{i j}
$$

where $A=\left[a_{i j}\right], B=\left[b_{k l}\right]$ are scalar matrices of order $m \times n$ and $p \times q$, respectively, $a_{i j} B$ is of order $p \times q$, and $A \otimes B$ of order $m p \times n q$;

Hindawi Publishing Corporation Journal of Inequalities and Applications Volume 2006, Article ID 80878, Pages 1-21 DOI $10.1155 /$ JIA/2006/80878 
2 Generalization inequalities for Khatri-Rao product

(ii) Hadamard product

$$
A \circ B=\left[a_{i j} b_{i j}\right]_{i j}=B \circ A,
$$

where $A=\left[a_{i j}\right], B=\left[b_{i j}\right]$ are scalar matrices of order $m \times n, a_{i j} b_{i j}$ is a scalar, and $A \circ B$ is of order $m \times n$;

(iii) Tracy-Singh product

$$
A \Theta B=\left[A_{i j} \Theta B\right]_{i j}=\left[\left[A_{i j} \otimes B_{k l}\right]_{k l}\right]_{i j}
$$

where $A=\left[A_{i j}\right], B=\left[B_{k l}\right]$ are partitioned matrices of order $m \times n$ and $p \times$ $q$, respectively, $A_{i j}$ is of order $m_{i} \times n_{j}, B_{k l}$ of order $p_{k} \times q_{l}, A_{i j} \otimes B_{k l}$ of order $m_{i} p_{k} \times n_{j} q_{l}, A_{i j} \Theta B$ of order $m_{i} p \times n_{j} q\left(m=\sum_{i=1}^{t} m_{i}, n=\sum_{j=1}^{d} n_{j}, p=\sum_{k=1}^{u} p_{k}\right.$, $\left.q=\sum_{l=1}^{v} q_{l}\right)$, and $A \Theta B$ of order $m p \times n q ;$

(iv) Khatri-Rao product

$$
A * B=\left[A_{i j} \otimes B_{i j}\right]_{i j}
$$

where $A=\left[A_{i j}\right], B=\left[B_{i j}\right]$ are partitioned matrices of order $m \times n$ and $p \times q$, respectively, $A_{i j}$ is of order $m_{i} \times n_{j}, B_{k l}$ of order $p_{i} \times q_{j}, A_{i j} \otimes B_{i j}$ of order $m_{i} p_{i} \times$ $n_{j} q_{j}\left(m=\sum_{i=1}^{t} m_{i}, n=\sum_{j=1}^{d} n_{j}, p=\sum_{i=1}^{t} p_{i}, q=\sum_{j=1}^{d} q_{j}\right)$, and $A * B$ of order $M \times N\left(M=\sum_{i=1}^{t} m_{i} p_{i}, N=\sum_{j=1}^{d} n_{j} q_{j}\right)$.

In general, $A \Theta B \neq B \Theta A, A \otimes B \neq B \otimes A, A * B \neq B * A$, but if $A=\left[a_{i j}\right]$ is a scalar matrix and $B=\left[B_{i j}\right]$ is a partitioned matrix, then $A * B=B * A$. Additionally, Liu [5] shows that the Khatri-Rao product can be viewed as a generalized Hadamard product and the Tracy-Singh product as a generalized Kronecker product, as follows:

(1) for a nonpartitioned matrix $A$, their $A \Theta B$ is $A \otimes B$, that is,

$$
A \Theta B=\left[a_{i j} \Theta B\right]_{i j}=\left[\left[a_{i j} \otimes B_{k l}\right]_{k l}\right]_{i j}=\left[\left[a_{i j} B_{k l}\right]_{k l}\right]_{i j}=\left[a_{i j} B\right]_{i j}=A \otimes B
$$

(2) for nonpartitioned matrices $A$ and $B$ of order $m \times n$, their $A * B$ is $A \circ B$, that is,

$$
A * B=\left[a_{i j} \otimes b_{i j}\right]_{i j}=\left[a_{i j} b_{i j}\right]_{i j}=A \circ B
$$

The Khatri-Rao and Tracy-Singh products are related by the following relation $[5,6]$ :

$$
A * B=Z_{1}^{T}(A \Theta B) Z_{2}
$$

where $A=\left[A_{i j}\right]$ is partitioned with $A_{i j}$ of order $m_{i} \times n_{j}$ and $B=\left[B_{k l}\right]$ is partitioned with $B_{k l}$ of order $p_{k} \times q_{l}\left(m=\sum_{i=1}^{t} m_{i}, n=\sum_{j=1}^{d} n_{j}, p=\sum_{k=1}^{u} p_{k}, q=\sum_{l=1}^{v} q_{l}\right), Z_{1}$ is an $m p \times$ $r\left(r=\sum_{i=1}^{t} m_{i} p_{i}\right)$ matrix of zeros and ones, and $Z_{2}$ is an $n q \times s\left(s=\sum_{j=1}^{d} n_{j} q_{j}\right)$ matrix 
of zeros and ones such that $Z_{1}^{T} Z_{1}=I_{r}, Z_{2}^{T} Z_{2}=I_{s}\left(I_{r}\right.$ and $I_{s}$ are $r \times r$ and $s \times s$ identity matrices, resp.).

In particular, if $m=n$ and $p=q$, then there exists an $m p \times r\left(r=\sum_{i=1}^{t} m_{i} p_{i}\right)$ matrix $Z$ such that $Z^{T} Z=I_{r}\left(I_{r}\right.$ is an $r \times r$ identity matrix $)$ and

$$
A * B=Z^{T}(A \Theta B) Z
$$

Here

$$
Z=\left[\begin{array}{lll}
Z_{1} & & \\
& \ddots & \\
& & Z_{t}
\end{array}\right]
$$

where each $Z_{i}=\left[\begin{array}{lllllll}0_{i 1} & \cdots & 0_{i i-1} & I_{m_{i} p_{i}} & 0_{i i+1} & \cdots & 0_{i t}\end{array}\right]^{T}$ is an real matrix of zeros and ones, and $0_{i k}$ is a $m_{i} p_{i} \times m_{i} p_{k}$ zero matrix for any $k \neq i$. Note also that $Z_{i}^{T} Z_{i}=I$ and

$$
Z_{i}^{T}\left(A_{i j} \Theta B\right) Z_{j}=Z_{i}^{T}\left(A_{i j} \otimes B_{k l}\right)_{k l} Z_{j}=A_{i j} \otimes B_{i j}, \quad i, j=1,2, \ldots, t .
$$

In [5-8], the authors proved a number of equalities and inequalities involving KhatriRao and Hadamard products of two matrices. Here we extend these results in three ways. First, we establish new attractive equalities and inequalities involving Khatri-Rao product of matrices. Second, we recover and develop some results of Visick, for example, [8, Theorem 11, page 54]. This does not follow simply from the work of Visick. Third, the results are extended to the case of Khatri-Rao products of any finite number of matrices. This result leads to inequalities involving Hadamard product, as a special case.

We use the following notations:

(i) $M_{m, n}$ - the set of all $m \times n$ matrices over the complex number field $\mathbb{C}$ and when $m=n$, we write $M_{m}$ instead of $M_{m, n}$;

(ii) $A^{T}, A^{*}, A^{+}, A^{-1}$-the transpose, conjugate transpose, Moore-Penrose inverse, and inverse of matrix $A$, respectively.

For Hermitian matrices $A$ and $B$, the relation $A>B$ means that $A-B>0$ is a positive definite and the relation $A \geq B$ means $A-B \geq 0$ is a positive semidefinite. Given a positive definite matrix $A$, its positive definite square root is denoted by $A^{1 / 2}$. We use the known fact "for positive definite matrices $A$ and $B$, the relation $A \geq B$ implies $A^{1 / 2} \geq B^{1 / 2}$ " which is called the Löwner-Heinz theorem.

\section{Some notations and preliminary results}

Let $A$ be a positive definite $m \times m$ matrix. The spectral decomposition of matrix $A$ assures that there exists a unitary matrix $U$ such that

$$
A=U^{*} D U=U^{*} \operatorname{diag}\left(\lambda_{i}\right) U, \quad U^{*} U=I_{m},
$$




\section{Generalization inequalities for Khatri-Rao product}

where $D=\operatorname{diag}\left(\lambda_{i}\right)=\operatorname{diag}\left(\lambda_{1}, \ldots, \lambda_{m}\right)$ is the diagonal matrix with diagonal entries $\lambda_{i}\left(\lambda_{i}\right.$ are the positive eigenvalues of $A$ ). For any real number $r, A^{r}$ is defined by

$$
A^{r}=U^{*} D^{r} U=U^{*} \operatorname{diag}\left(\lambda_{i}^{r}\right) U
$$

If $A \in M_{m, n}$ is any matrix with $\operatorname{rank}(\mathrm{A})=s$, the singular value decomposition of $A$ assures that there are unitary matrices $U \in M_{m}$ and $V \in M_{n}$ such that

$$
A=U \sum V^{*}
$$

Here $\sum=\left[\begin{array}{cc}W & 0 \\ 0 & 0\end{array}\right] \in M_{m, n}$, where $W=\operatorname{diag}\left(\varsigma_{1}, \ldots, \varsigma_{s}\right) \in M_{s}$ is the diagonal matrix with diagonal entries $\varsigma_{i}(i=1,2, \ldots, s)$ and $\varsigma_{1} \geq \varsigma_{2} \geq \cdots \geq \varsigma_{s}>0$ are the singular values of $A$, that is, $\varsigma_{1} \geq \varsigma_{2} \geq \cdots \geq \varsigma_{s}>0$ are positive square roots of positive eigenvalues of $A^{*} A$ and $A A^{*}$. The Moore-Penrose inverse of $A$ is defined by

$$
A^{+}=V\left[\begin{array}{cc}
W^{-1} & 0 \\
0 & 0
\end{array}\right] U^{*} \in M_{n, m},
$$

where $W^{-1}=\operatorname{diag}\left(\varsigma_{1}^{-1}, \varsigma_{2}^{-1}, \ldots, \varsigma_{s}^{-1}\right) \in M_{s}$ is the diagonal matrix with diagonal entries $\varsigma_{i}^{-1}(i=1,2, \ldots, s) \cdot A^{+}$is a unique matrix which satisfies the following conditions:

$$
A A^{+} A=A, \quad A^{+} A A^{+}=A^{+}, \quad\left(A A^{+}\right)^{*}=A A^{+}, \quad\left(A^{+} A\right)^{*}=A^{+} A .
$$

For any compatible partitioned matrices $A, B, C$, and $D$, we will make a frequent use of the following properties of the Tracy-Singh product (see e.g., $[1,3,5,10]$ ):

(a) $(A \Theta B)(C \Theta D)=(A C) \Theta(B D)$ if $A C$ and $B D$ are well defined;

(b) $(A \Theta B)^{r}=A^{r} \Theta B^{r}$ if $A \in M_{m}, B \in M_{n}$ are positive semidefinite matrices and $r$ is any real number;

(c) $(A \Theta B)^{*}=A^{*} \Theta B^{*}$;

(d) $(A \Theta B)^{+}=A^{+} \Theta B^{+}$.

If $A \in M_{m}$ and $B \in M_{n}$ are positive semidefinite matrices, then (see, [3, 10])

(e) $A \Theta B \geq 0$;

(f) $\lambda_{1}(A \Theta B)=\lambda_{1}(A) \lambda_{1}(B), \lambda_{m n}(A \Theta B)=\lambda_{m}(A) \lambda_{n}(B)$,

where $\lambda_{1}(A), \lambda_{m}(A)$ are the largest and smallest eigenvalues, respectively, of a matrix $A$, and $\lambda_{1}(B), \lambda_{n}(B)$ are the largest and smallest eigenvalues, respectively, of a matrix $B$.

The Khatri-Rao and Tracy-Singh products of $k$ matrices $A_{i}(1 \leq i \leq k, k \geq 2)$ will be denoted by $\prod_{i=1}^{k} * A_{i}=A_{1} * A_{2} * \cdots * A_{k}$ and $\prod_{i=1}^{k} \Theta A_{i}=A_{1} \Theta A_{2} \Theta \cdots \Theta A_{k}$, respectively.

For a finite number of matrices $A_{i}(i=1,2, \ldots, k)$, the properties (a)-(d) become as in Lemma 2.1 and the connection between the Khatri-Rao and Tracy-Singh products in (1.7) and (1.8) becomes as in Lemma 2.2. 
Lemma 2.1. Let $A_{i}$ and $B_{i}(1 \leq i \leq k, k \geq 2)$ be compatible partitioned matrices. Then

(i)

$$
\left(\prod_{i=1}^{k} \Theta A_{i}\right)\left(\prod_{i=1}^{k} \Theta B_{i}\right)=\left(\prod_{i=1}^{k} \Theta\left(A_{i} B_{i}\right)\right)
$$

if $A_{i} B_{i}(1 \leq i \leq k, k \geq 2)$ are well defined;

(ii)

$$
\left(\prod_{i=1}^{k} \Theta A_{i}\right)^{+}=\prod_{i=1}^{k} \Theta A_{i}^{+}, \quad k=2,3, \ldots
$$

(iii)

$$
\left(\prod_{i=1}^{k} \Theta A_{i}\right)^{*}=\prod_{i=1}^{k} \Theta A_{i}^{*}, \quad\left(\prod_{i=1}^{k} * A_{i}\right)^{*}=\prod_{i=1}^{k} * A_{i}^{*}, \quad k=2,3, \ldots
$$

(iv)

$$
\left(\prod_{i=1}^{k} \Theta A_{i}\right)^{r}=\prod_{i=1}^{k} \Theta A_{i}^{r} \quad \text { if } A_{i} \in M_{m(i)}(1 \leq i \leq k, k \geq 2)
$$

are positive semidefinite matrices and $r$ is any real number;

(v)

$$
\left(\prod_{i=1}^{k}\left(A_{i} \Theta B_{i}\right)\right)=\left(\prod_{i=1}^{k} A_{i}\right) \Theta\left(\prod_{i=1}^{k} B_{i}\right), \quad k=2,3, \ldots
$$

Proof. The proof is immediately derived by induction on $k$.

Lemma 2.2. Let $A_{i}=\left[A_{g h}^{(i)}\right] \in M_{m(i), n(i)}(1 \leq i \leq k, k \geq 2)$ be partitioned matrices with $A_{g h}^{(i)}$ as the $(g, h)$ th block submatrix $\left(m=\prod_{i=1}^{k} m(i), n=\prod_{i=1}^{k} n(i), r=\sum_{j=1}^{t} \prod_{i=1}^{k} m_{j}(i)\right.$, $\left.s=\sum_{j=1}^{t} \prod_{i=1}^{k} n_{j}(i), m(i)=\sum_{j=1}^{t} m_{j}(i), n(i)=\sum_{j=1}^{t} n_{j}(i)\right)$. Then there exist two real $m a-$ trices $Z_{1}$ of order $m \times r$ and $Z_{2}$ of order $n \times$ s such that $Z_{1}^{T} Z_{1}=I_{r}, Z_{2}^{T} Z_{2}=I_{s}\left(Z_{1}, Z_{2}\right.$ are real matrices of zeros and ones) and

$$
\prod_{i=1}^{k} * A_{i}=Z_{1}^{T}\left(\prod_{i=1}^{k} \Theta A_{i}\right) Z_{2}, \quad k=2,3, \ldots
$$

where $I_{r}$ and $I_{s}$ are identity matrices of order $r \times r$ and $s \times s$, respectively. In particular, if 
6 Generalization inequalities for Khatri-Rao product

$m(i)=n(i)(1 \leq i \leq k, k \geq 2)$, then there exists an $m \times r$ matrix $Z$ of zeros and ones such that $Z^{T} Z=I_{r}$,

$$
\prod_{i=1}^{k} * A_{i}=Z^{T}\left(\prod_{i=1}^{k} \Theta A_{i}\right) Z, \quad k=2,3, \ldots
$$

and $Z Z^{T}$ is an $m \times m$ diagonal matrix of zeros and ones, so

$$
0 \leq Z Z^{T} \leq I_{m}
$$

where $m=\prod_{i=1}^{k} m(i)$.

Proof. The special case in (2.12) of Lemma 2.2 is proved in [3, Corollary 2.2] and (2.13) follows immediately by the definition of matrix $Z$. We give proof of the general case in (2.11) of Lemma 2.2 for the sake of convenience. We proceed by induction on $k$. If $k=2$, then (2.11) is true by (1.7). Now suppose (2.11) holds for the Khatri-Rao product of $k$ matrices, that is, there exist an $m \times r$ matrix $P_{k r}$ of zeros and ones and an $n \times s$ matrix $R_{k s}$ of zeros and ones such that $P_{k r}^{T} P_{k r}=I_{r}, R_{k s}^{T} R_{k s}=I_{s}$, and

$$
\prod_{i=1}^{k} * A_{i}=P_{k r}^{T}\left(\prod_{i=1}^{k} \Theta A_{i}\right) R_{k s}, \quad k=2,3, \ldots
$$

We will prove that it is true for the Khatri-Rao product of $k+1$ matrices. Then by (1.7), there exist an $m(1) r \times r$ matrix $Q_{1}$ of zeros and ones and an $n(1) s \times s$ matrix $Q_{2}$ of zeros and ones such that $Q_{1}^{T} Q_{1}=I_{r}, Q_{2}^{T} Q_{2}=I_{s}$, and

$$
\begin{aligned}
\prod_{i=1}^{k+1} * A_{i} & =A_{1} *\left(\prod_{i=2}^{k+1} * A_{i}\right)=Q_{1}^{T}\left(A_{1} \Theta \prod_{i=2}^{k+1} * A_{i}\right) Q_{2}=Q_{1}^{T}\left\{A_{1} \Theta\left(P_{k r}^{T}\left(\prod_{i=2}^{k+1} \Theta A_{i}\right) R_{k s}\right)\right\} Q_{2} \\
& =Q_{1}^{T}\left\{\left(I_{m(1)} A_{1} I_{n(1)}\right) \Theta\left(P_{k r}^{T}\left(\prod_{i=2}^{k+1} \Theta A_{i}\right) R_{k s}\right)\right\} Q_{2} \\
& =Q_{1}^{T}\left(I_{m(1)} \Theta P_{k r}^{T}\right)\left\{A_{1} \Theta\left(\prod_{i=2}^{k+1} \Theta A_{i}\right)\right\}\left(I_{n(1)} \Theta R_{k s}\right) Q_{2} \\
& =Q_{1}^{T}\left(I_{m(1)} \Theta P_{k r}^{T}\right)\left(\prod_{i=1}^{k+1} \Theta A_{i}\right)\left(I_{n(1)} \Theta R_{k s}\right) Q_{2} .
\end{aligned}
$$

Letting $Z_{1}=\left(I_{m(1)} \Theta P_{k r}\right) Q_{1}$ and $Z_{2}=\left(I_{n(1)} \Theta R_{k s}\right) Q_{2}$, the inductive step is complete. Here $Q_{1}=P_{2 r}=P_{r}, Q_{1}=R_{2 s}=R_{s}$, and it is a simple matter to verify that

$$
\begin{array}{cc}
Z_{1}=\left(I_{m(1)} \Theta P_{k r}\right) P_{r}=P_{(k+1) r}, & Z_{1}^{T}=P_{r}^{T}\left(I_{m(1)} \Theta P_{k r}^{T}\right)=P_{(k+1) r}^{T}, \\
Z_{2}=\left(I_{n(1)} \Theta R_{k s}\right) R_{s}=R_{(k+1) s}, & Z_{2}^{T}=R_{s}^{T}\left(I_{n(1)} \Theta R_{k s}^{T}\right)=R_{(k+1) s}^{T} .
\end{array}
$$


Note that

$$
\begin{aligned}
Z_{1}^{T} Z_{1} & =P_{r}^{T}\left(I_{m(1)} \Theta P_{k r}^{T}\right)\left(I_{m(1)} \Theta P_{k r}\right) P_{r}=Q_{1}^{T}\left(I_{m(1)} \Theta P_{k r}^{T}\right)\left(I_{m(1)} \Theta P_{k r}\right) Q_{1} \\
& =Q_{1}^{T}\left(I_{m(1)} I_{m(1)} \Theta P_{k r}^{T} P_{k r}\right) Q_{1} \\
& =Q_{1}^{T}\left(I_{m(1)} \Theta I_{r}\right) Q_{1} \quad\left(I_{m(1)} \Theta I_{r}=I_{m(1) r}\right) \\
& =Q_{1}^{T}\left(I_{m(1) r}\right) Q_{1}=Q_{1}^{T} Q_{1}=I_{r} .
\end{aligned}
$$

Similarly, it is easy to verify that $Z_{2}^{T} Z_{2}=I_{s}$.

Lemma 2.3. Let $\alpha$ be a nonempty subset of the set $\{1,2, \ldots, m\}$ and let $A \in M_{m}$ be a positive semidefinite matrix. Then (see Chollet [4])

(i) if either $-1 \leq r \leq 0$ or $1 \leq r \leq 2$, then

$$
A^{r}(\alpha) \geq A(\alpha)^{r}, \quad \forall \alpha
$$

(ii) if $0 \leq r \leq 1$, then

$$
A^{r}(\alpha) \leq A(\alpha)^{r}, \quad \forall \alpha,
$$

where $A(\alpha)$ is the principal submatrix of $A$ whose entries are in the intersection of the rows and columns of A specified by $\alpha$.

Lemma 2.4. Let $X_{j}>0(j=1,2, \ldots, k)$ be $n \times n$ matrices with eigenvalues in the interval $[w, W]$ and $U_{j}(j=1,2, \ldots, k)$ are $r \times m$ matrices such that $\sum_{j=1}^{k} U_{j} U_{j}^{*}=I$. Then (see Mond and Pečarić [7])

(i) for every real $p>1$ and $p<0$,

$$
\sum_{j=1}^{k} U_{j} X_{j}^{p} U_{j}^{*} \leq \mu\left\{\sum_{j=1}^{k} U_{j} X_{j} U_{j}^{*}\right\}^{p},
$$

where

$$
\mu=\frac{\delta^{p}-\delta}{(p-1)(\delta-1)}\left(\frac{p-1}{p} \frac{\delta^{p}-1}{\delta^{p}-\delta}\right)^{p}, \quad \delta=\frac{W}{w} .
$$

While for $0<p<1$, the reverse inequality holds in (2.20);

(ii) for every real $p>1$ and $p<0$,

$$
\left\{\sum_{j=1}^{k} U_{j} X_{j}^{p} U_{j}^{*}\right\}-\left\{\sum_{j=1}^{k} U_{j} X_{j} U_{j}^{*}\right\}^{p} \leq \gamma\{I\},
$$

where

$$
\gamma=\frac{W w^{p}-w W^{p}}{W-w}+(p-1)\left(\frac{1}{p} \frac{W^{p}-w^{P}}{W-w}\right)^{p /(p-1)} .
$$

While for $0<p<1$, the reverse inequality holds in (2.22). 
8 Generalization inequalities for Khatri-Rao product

\section{New applications and results}

Based on the basic results in Section 2 and the general connection between the Khatri-Rao and Tracy-Singh products in Lemma 2.2, we generalize and derive some equalities and inequalities in works of Visick [8, Corollary 3, Theorem 4], Chollet [4], and Mond and Pečarić [7] with respect to the Khatri-Rao product and extend these results to any finite number of matrices. These results lead to inequalities involving Hadamard products, as a special case.

Theorem 3.1. Let $A_{i}=\left[A_{g h}^{(i)}\right] \in M_{m(i), n(i)}(1 \leq i \leq k, k \geq 2)$ be partitioned matrices with $A_{g h}^{(i)}$ as the $(g, h)$ th block submatrix $\left(m=\prod_{i=1}^{k} m(i), n=\prod_{i=1}^{k} n(i)\right)$ and let $Z_{1}$ and $Z_{2}$ be the real matrices of zeros and ones that satisfy (2.11). Then

(i) there exists an $m \times(m-r)$ matrix $Q_{(m)}$ of zeros and ones such that the block matrix $\Omega=\left[Z_{1} Q_{(m)}\right]$ is an $m \times m$ permutation matrix. $Q_{(m)}$ is not unique but for any such choice of $Q_{(m)}$,

$$
Z_{1}^{T} Q_{(m)}=0, \quad Q_{(m)}^{T} Q_{(m)}=I_{m-r}, \quad Q_{(m)} Q_{(m)}^{T}+Z_{1} Z_{1}^{T}=I_{m}
$$

(ii) for any $m \times n$ matrix $L$,

$$
Z_{1}^{T} L L^{*} Z_{1} \geq\left(Z_{1}^{T} L Z_{2}\right)\left(Z_{1}^{T} L Z_{2}\right)^{*} \geq 0
$$

Proof. Though the proof is quite similar to the proof of [8, Corollary 3(iii) and (vii)] for Hadamard product, we give proof for the sake of convenience.

(i) It is evident from the structure of $Z_{1}$ that it may be considered as part of an $m \times m$ permutation matrix $\Omega=\left[Z_{1} Q_{(m)}\right]$, where $Q_{(m)}$ is an $m \times(m-r)$ matrix of zeros and ones. For example, when $k=2$, then $Q_{(2)}$ is not unique (see, [8, page 49]). Using the properties of a permutation matrix together with the definition of $\Omega=\left[Z_{1} Q_{(m)}\right]$, we have

$$
\begin{gathered}
I_{m}=\Omega \Omega^{T}=\left[\begin{array}{ll}
Z_{1} & Q_{(m)}
\end{array}\right]\left[\begin{array}{c}
Z_{1}^{T} \\
Q_{(m)}^{T}
\end{array}\right]=Q_{(m)} Q_{(m)}^{T}+Z_{1} Z_{1}^{T} \\
I_{m}=\left[\begin{array}{cc}
I_{r} & 0 \\
0 & I_{m-r}
\end{array}\right]=\Omega^{T} \Omega=\left[\begin{array}{c}
Z_{1}^{T} \\
Q_{m}^{T}
\end{array}\right]\left[\begin{array}{ll}
Z_{1} & Q_{(m)}
\end{array}\right]=\left[\begin{array}{cc}
Z_{1}^{T} Z_{1} & Z_{1}^{T} Q_{(m)} \\
Q_{(m)}^{T} Z_{1} & Q_{(m)}^{T} Q_{(m)}
\end{array}\right] .
\end{gathered}
$$

From these come the required results in (i), that is,

$$
Z_{1}^{T} Q_{(m)}=0, \quad Q_{(m)}^{T} Q_{(m)}=I_{m-r}, \quad Q_{(m)} Q_{(m)}^{T}+Z_{1} Z_{1}^{T}=I_{m}
$$

(ii) By (2.13) of Lemma 2.2, we have $I_{n} \geq Z_{2} Z_{2}^{T} \geq 0$ and so

$$
Z_{1}^{T} L L^{*} Z_{1} \geq Z_{1}^{T} L Z_{2} Z_{2}^{T} L^{*} Z_{1}=\left(Z_{1}^{T} L Z_{2}\right)\left(Z_{1}^{T} L Z_{2}\right)^{*} \geq 0
$$

We now generalize [8, Theorem 4$]$ to the case of Khatri-Rao product involving a finite number of matrices. 
Theorem 3.2. Let $A_{i}=\left[A_{g h}^{(i)}\right] \in M_{m, n}(1 \leq i \leq k, k \geq 2)$ be partitioned matrices with $A_{g h}^{(i)}$ as the $(g, h)$ th block submatrix. Let $Z_{1}$ be an $m^{k} \times r$ matrix of zeros and ones that satisfies (2.12) and let $Q_{(n)}$ be an $n^{k} \times\left(n^{k}-s\right)$ matrix of zeros and ones that satisfies (3.1). Then

$$
\begin{aligned}
\prod_{i=1}^{k} *\left(A_{i} A_{i}^{*}\right) & =\left(\prod_{i=1}^{k} *\left(A_{i}\right)\right)\left(\prod_{i=1}^{k} * A_{i}\right)^{*}+Z_{1}^{T}\left(\prod_{i=1}^{k} \Theta A_{i}\right) Q_{(n)} Q_{(n)}^{T}\left(\prod_{i=1}^{k} \Theta A_{i}\right)^{*} Z_{1} \\
& =\left(\prod_{i=1}^{k} *\left(A_{i}\right)\right)\left(\prod_{i=1}^{k} * A_{i}\right)^{*}+\left(Z_{1}^{T}\left(\prod_{i=1}^{k} \Theta A_{i}\right) Q_{(n)}\right)\left(Z_{1}^{T}\left(\prod_{i=1}^{k} \Theta A_{i}\right) Q_{(n)}\right)^{*},
\end{aligned}
$$

and hence

$$
\prod_{i=1}^{k} *\left(A_{i} A_{i}^{*}\right) \geq\left(\prod_{i=1}^{k} *\left(A_{i}\right)\right)\left(\prod_{i=1}^{k} * A_{i}\right)^{*}, \quad k=2,3, \ldots
$$

Proof. From Lemma 2.1(i) and (iii), we have

$$
\prod_{i=1}^{k} \Theta\left(A_{i} A_{i}^{*}\right)=\left(\prod_{i=1}^{k} \Theta A_{i}\right)\left(\prod_{i=1}^{k} \Theta A_{i}\right)^{*} .
$$

But by Theorem 3.1(i), there exist an $n^{k} \times s$ matrix $Z_{2}$ of zeros and ones that satisfies (2.12) and an $n^{k} \times\left(n^{k}-s\right)$ matrix $Q_{(n)}$ of zeros and ones that satisfies (3.1) such that $Z_{2} Z_{2}^{T}+Q_{(n)} Q_{(n)}^{T}=I_{n^{k}}$ and

$$
\begin{aligned}
\prod_{i=1}^{k} \Theta\left(A_{i} A_{i}^{*}\right) & =\left(\prod_{i=1}^{k} \Theta A_{i}\right)\left(Z_{2} Z_{2}^{T}+Q_{(n)} Q_{(n)}^{T}\right)\left(\prod_{i=1}^{k} \Theta A_{i}\right)^{*} \\
& =\left(\prod_{i=1}^{k} \Theta A_{i}\right)\left(Z_{2} Z_{2}^{T}\right)\left(\prod_{i=1}^{k} \Theta A_{i}\right)^{*}+\left(\prod_{i=1}^{k} \Theta A_{i}\right)\left(Q_{(n)} Q_{(n)}^{T}\right)\left(\prod_{i=1}^{k} \Theta A_{i}\right)^{*} .
\end{aligned}
$$

Since $A_{i}(1 \leq i \leq k, k \geq 2)$ are rectangular partitioned matrices of order $m \times n$, then due to (2.11) of Lemma 2.2 there exist two real matrices $Z_{1}$ and $Z_{2}$ of zeros and ones of order $m^{k} \times r$ and $n^{k} \times s$, respectively, such that

$$
\prod_{i=1}^{k} * A_{i}=Z_{1}^{T}\left(\prod_{i=1}^{k} \Theta A_{i}\right) Z_{2}, \quad k=2,3, \ldots
$$

But because $A_{i} A_{i}^{*} \quad(1 \leq i \leq k, k \geq 2)$ are square matrices of order $m \times m$, then due to (2.12) of Lemma 2.2 there exists a real matrix $Z_{1}$ of zeros and ones of order $m^{k} \times r$ such that

$$
\prod_{i=1}^{k} *\left(A_{i} A_{i}^{*}\right)=Z_{1}^{T}\left(\prod_{i=1}^{k} \Theta\left(A_{i} A_{i}^{*}\right)\right) Z_{1}, \quad k=2,3, \ldots
$$


10 Generalization inequalities for Khatri-Rao product

Due to (3.9), (3.10), and (3.11), we have

$$
\begin{aligned}
\prod_{i=1}^{k} *\left(A_{i} A_{i}^{*}\right)= & Z_{1}^{T}\left(\prod_{i=1}^{k} \Theta\left(A_{i} A_{i}^{*}\right)\right) Z_{1}=Z_{1}^{T}\left(\prod_{i=1}^{k} \Theta A_{i}\right) Z_{2} Z_{2}^{T}\left(\prod_{i=1}^{k} \Theta A_{i}\right)^{*} Z_{1} \\
& +Z_{1}^{T}\left(\prod_{i=1}^{k} \Theta A_{i}\right)\left(Q_{(n)} Q_{(n)}^{T}\right)\left(\prod_{i=1}^{k} \Theta A_{i}\right)^{*} Z_{1} \\
= & \left(Z_{1}^{T}\left(\prod_{i=1}^{k} \Theta A_{i}\right) Z_{2}\right)\left(Z_{1}^{T}\left(\prod_{i=1}^{k} \Theta A_{i}\right) Z_{2}\right)^{*} \\
& +Z_{1}^{T}\left(\prod_{i=1}^{k} \Theta A_{i}\right)\left(Q_{(n)} Q_{(n)}^{T}\right)\left(\prod_{i=1}^{k} \Theta A_{i}\right)^{*} Z_{1} \\
= & \left(\prod_{i=1}^{k} *\left(A_{i}\right)\right)\left(\prod_{i=1}^{k} * A_{i}\right)^{*}+Z_{1}^{T}\left(\prod_{i=1}^{k} \Theta A_{i}\right) Q_{(n)} Q_{(n)}^{T}\left(\prod_{i=1}^{k} \Theta A_{i}\right)^{*} Z_{1} \\
= & \left(\prod_{i=1}^{k} *\left(A_{i}\right)\right)\left(\prod_{i=1}^{k} * A_{i}\right)^{*}+\left(Z_{1}^{T}\left(\prod_{i=1}^{k} \Theta A_{i}\right) Q_{(n)}\right)\left(Z_{1}^{T}\left(\prod_{i=1}^{k} \Theta A_{i}\right) Q_{(n)}\right)^{*} .
\end{aligned}
$$

If we put $k=2$ in Theorem 3.2, we obtain the following corollary.

Corollary 3.3. Let $A_{i}=\left[A_{g h}^{(i)}\right] \in M_{m, n}(1 \leq i \leq 2)$ be partitioned matrices with $A_{g h}^{(i)}$ as the $(g, h)$ th block submatrix. Let $Z_{1}$ be an $m^{2} \times r$ matrix of zeros and ones that satisfies (1.8) and let $Q_{(n)}$ be an $n^{2} \times\left(n^{2}-s\right)$ matrix of zeros and ones that satisfies (3.1). Then

$$
A_{1} A_{1}^{*} * A_{2} A_{2}^{*}=\left(A_{1} * A_{2}\right)\left(A_{1} * A_{2}\right)^{*}+Z_{1}^{T}\left(A_{1} \Theta A_{2}\right) Q_{(n)} Q_{(n)}^{T}\left(A_{1} \Theta A_{2}\right)^{*} Z_{1},
$$

and hence

$$
A_{1} A_{1}^{*} * A_{2} A_{2}^{*} \geq\left(A_{1} * A_{2}\right)\left(A_{1} * A_{2}\right)^{*} .
$$

Corollary 3.4. Let $A_{i}=\left[A_{g h}^{(i)}\right] \in M_{m, n}(1 \leq i \leq k, k \geq 2)$ be partitioned matrices with $A_{g h}^{(i)}$ as the $(g, h)$ th block submatrix. Let $Z_{1}$ be an $m^{k} \times r$ matrix of zeros and ones that satisfies (2.12) and let $Q_{(n)}$ be an $n^{k} \times\left(n^{k}-s\right)$ matrix of zeros and ones that satisfies (3.1). Then the following statements are equivalent:

(i)

$$
\prod_{i=1}^{k} *\left(A_{i} A_{i}^{*}\right)=\left(\prod_{i=1}^{k} *\left(A_{i}\right)\right)\left(\prod_{i=1}^{k} * A_{i}\right)^{*}, \quad k=2,3, \ldots
$$

(ii)

$$
Z_{1}^{T}\left(\prod_{i=1}^{k} \Theta A_{i}\right) Q_{(n)}=0, \quad k=2,3, \ldots ;
$$


(iii)

$$
\prod_{i=1}^{k} *\left(A_{i} X_{i}\right)=\left(\prod_{i=1}^{k} *\left(A_{i}\right)\right)\left(\prod_{i=1}^{k} *\left(X_{i}\right)\right), \quad \text { for } X_{i} \in M_{n, m}(1 \leq i \leq k, k \geq 2) .
$$

Proof. To arrive from (i) to (ii), notice that (i) holds if and only if the last term of (3.6) is zero, which is equivalent to $Z_{1}^{T}\left(\prod_{i=1}^{k} \Theta A_{i}\right) Q_{(n)}=0$. To arrive from (ii) to (iii), notice that (ii) may be rewritten as $Z_{1}^{T}\left(\prod_{i=1}^{k} \Theta A_{i}\right) Q_{(n)} Q_{(n)}^{T}=0$. By Theorem 3.1(i), there exist an $n^{k} \times s$ matrix $Z_{2}$ of zeros and ones that satisfies (2.12) and an $n^{k} \times\left(n^{k}-s\right)$ matrix $Q_{(n)}$ of zeros and ones that satisfies (3.1) such that $Q_{(n)} Q_{(n)}^{T}=I_{n^{k}}-Z_{2} Z_{2}^{T}$, this becomes

$$
Z_{1}^{T}\left(\prod_{i=1}^{k} \Theta A_{i}\right)=Z_{1}^{T}\left(\prod_{i=1}^{k} \Theta A_{i}\right) Z_{2} Z_{2}^{T}
$$

By postmultiplying by $\left(\prod_{i=1}^{k} \Theta X_{i}\right) Z_{1}$ for any of the $n \times m$ matrices $X_{i}(1 \leq i \leq k)$, we have

$$
Z_{1}^{T}\left(\prod_{i=1}^{k} \Theta\left(A_{i} X_{i}\right)\right) Z_{1}=Z_{1}^{T}\left(\prod_{i=1}^{k} \Theta A_{i}\right) Z_{2} Z_{2}^{T}\left(\prod_{i=1}^{k} \Theta X_{i}\right) Z_{1}
$$

which is (iii) by (2.11) and (2.12) of Lemma 2.2. To arrive from (iii) to (i), assume (iii) holds for all $n \times m$ matrices $X_{i}(1 \leq i \leq k)$. It must therefore be true for $X_{i}=A_{i}^{*}(1 \leq i \leq$ $k$ ), which is condition (i). Hence (iii) implies (3.6) which is (i).

If we put $k=2$ in Corollary 3.4, we obtain the following corollary.

COROLlary 3.5. Let $A_{i}=\left[A_{g h}^{(i)}\right] \in M_{m, n}(1 \leq i \leq 2)$ be partitioned matrices with $A_{g h}^{(i)}$ as the $(g, h)$ th block submatrix. Let $Z_{1}$ be an $m^{2} \times r$ matrix of zeros and ones that satisfies (1.8) and let $Q_{(n)}$ be an $n^{2} \times\left(n^{2}-s\right)$ matrix of zeros and ones that satisfies (3.1). Then the following statements are equivalent:

(i)

$$
A_{1} A_{1}^{*} * A_{2} A_{2}^{*}=\left(A_{1} * A_{2}\right)\left(A_{1} * A_{2}\right)^{*}
$$

(ii)

$$
Z_{1}^{T}\left(A_{1} \Theta A_{2}\right) Q_{(n)}=0
$$

(iii)

$$
A_{1} X_{1} * A_{2} X_{2}=\left(A_{1} * A_{2}\right)\left(X_{1} * X_{2}\right), \quad \text { for } X_{1}, X_{2} \in M_{n, m} \text {. }
$$

Theorem 3.6. Let $A_{i} \geq 0(1 \leq i \leq k, k \geq 2)$ be $n \times n$ compatible partitioned matrices. Then

(i) if either $-1 \leq r \leq 0$ or $1 \leq r \leq 2$, then

$$
\prod_{i=1}^{k} * A_{i}^{r} \geq\left(\prod_{i=1}^{k} * A_{i}\right)^{r}
$$


12 Generalization inequalities for Khatri-Rao product

(ii) if $0 \leq r \leq 1$, then

$$
\prod_{i=1}^{k} * A_{i}^{r} \leq\left(\prod_{i=1}^{k} * A_{i}\right)^{r} .
$$

Proof. If we put $s=1$, replace $r$ by $1 / r$ and $A_{i}$ by $A_{i}^{r}$ in [3, Theorem 3.1(i)], we obtain (i). But, if we put $s=-1$, replace $r$ by $1 /-r$ and $A_{i}$ by $A_{i}^{-r}$ in [3, Theorem 3.1(i)], we obtain (ii).

Remark 3.7. It is easy to give another proof of Theorem 3.6 by replacing $A$ by $\prod_{i=1}^{k} \Theta A_{i}$ in Lemma 2.3 and applying (2.12) of Lemma 2.2.

Theorem 3.8. Let $A_{i}>0$ be compatible partitioned matrices such that $\prod_{i=1}^{k} \Theta A_{i}>0(1 \leq$ $i \leq k, k \geq 2)$. Let $W$ and $w$ be the largest and smallest eigenvalues of $\prod_{i=1}^{k} \Theta A_{i}$, respectively. Then

(i) for every real $p>1$ and $p<0$,

$$
\prod_{i=1}^{k} * A_{i}^{p} \leq \mu\left(\prod_{i=1}^{k} * A_{i}\right)^{p}, \quad k=2,3, \ldots
$$

where

$$
\mu=\frac{\delta^{p}-\delta}{(p-1)(\delta-1)}\left(\frac{p-1}{p} \frac{\delta^{p}-1}{\delta^{p}-\delta}\right)^{p}, \quad \delta=\frac{W}{w} .
$$

While for every $0<p<1$, the reverse inequality holds in (3.25);

(ii) for every real $p>1$ and $p<0$,

$$
\prod_{i=1}^{k} * A_{i}^{p}-\left(\prod_{i=1}^{k} * A_{i}\right)^{p} \leq \gamma I, \quad k=2,3, \ldots,
$$

where

$$
\gamma=\frac{W w^{p}-w W^{p}}{W-w}+(p-1)\left(\frac{1}{p} \frac{W^{p}-w^{P}}{W-w}\right)^{p /(p-1)} .
$$

While for every $0<p<1$, the reverse inequality holds in (3.27).

Proof. This theorem follows from [3, Theorem 3.1(ii) and (iii)]. We give proof for the sake of convenience. In (2.20) and (2.22) of Lemma 2.4, set $k=1$ and replace $U$ by $Z^{T}$, $U^{*}$ by $Z$, and $X$ by $\prod_{i=1}^{k} \Theta A_{i}$, where $Z$, is the selection matrix of zeros and ones that satisfies (2.12). By using Lemma 2.1(iv), we establish Theorem 3.8.

From (3.25), we have the following special cases:

(i) for $p=2$, we have

$$
\prod_{i=1}^{k} * A_{i}^{2} \leq\left\{\frac{(W+w)^{2}}{4 w W}\right\}\left(\prod_{i=1}^{k} * A_{i}\right)^{2}, \quad k=2,3, \ldots ;
$$


(ii) for $p=-1$, we have

$$
\prod_{i=1}^{k} * A_{i}^{-1} \leq\left\{\frac{(W+w)^{2}}{4 w W}\right\}\left(\prod_{i=1}^{k} * A_{i}\right)^{-1}, \quad k=2,3, \ldots
$$

From (3.27), we have the following special cases:

(i) for $p=2$, we have

$$
\prod_{i=1}^{k} * A_{i}^{2}-\left(\prod_{i=1}^{k} * A_{i}\right)^{2} \leq \frac{1}{4}(W-w)^{2}\{I\}, \quad k=2,3, \ldots
$$

(ii) for $p=-1$, we have

$$
\prod_{i=1}^{k} * A_{i}^{-1}-\left(\prod_{i=1}^{k} * A_{i}\right)^{-1} \leq\left\{\frac{\sqrt{W}-\sqrt{w}}{w W}\right\} I, \quad k=2,3, \ldots
$$

\section{Further developments and applications}

Due to Albert's theorem in [2] and [9, Theorem 6.13], for a partitioned matrix $\left[\begin{array}{cc}A & B \\ B^{*} & D\end{array}\right]$ with a positive (semi) definite matrix $A \in M_{m}$,

$$
\left[\begin{array}{cc}
A & B \\
B^{*} & D
\end{array}\right] \geq 0 \quad \text { iff } D \geq B^{*} A^{+} B
$$

for any positive semidefinite matrix $D \in M_{n}$. It is also known that if matrix $A$ is square and nonsingular, then $A^{+}=A^{-1}$ and $\left[\begin{array}{cc}A & B \\ B^{*} & D\end{array}\right] \geq 0$ if and only if $D \geq B^{*} A^{-1} B$.

Let $Z_{1}$ and $Z_{2}$ be the real matrices of zeros and ones of order $m \times r$ and $n \times s$, respectively, that satisfy (2.11) in Lemma 2.2. Now another way to use Lemma 2.2 to generate inequalities involving the Khatri-Rao product is by using the following obvious inequality:

$$
T T^{*}=\left[\begin{array}{c}
T_{1} \\
T_{2}
\end{array}\right]\left[\begin{array}{ll}
T_{1}^{*} & T_{2}^{*}
\end{array}\right]=\left[\begin{array}{ll}
T_{1} T_{1}^{*} & T_{1} T_{2}^{*} \\
T_{2} T_{1}^{*} & T_{2} T_{2}^{*}
\end{array}\right] \geq 0
$$

where $T_{1}$ and $T_{2}$ are $n \times l$ and $m \times l$ matrices, respectively. Note that $T_{1} T_{1}^{*}$ and $T_{2} T_{2}^{*}$ are positive semidefinite (positive definite) matrices for every (nonsingular) complex matrices $T_{1}$ and $T_{2}$. This leads to

$$
\left[\begin{array}{cc}
Z_{2}^{T} & 0 \\
0 & Z_{1}^{T}
\end{array}\right]\left[\begin{array}{cc}
T_{1} T_{1}^{*} & T_{1} T_{2}^{*} \\
T_{2} T_{1}^{*} & T_{2} T_{2}^{*}
\end{array}\right]\left[\begin{array}{cc}
Z_{2} & 0 \\
0 & Z_{1}
\end{array}\right]=\left[\begin{array}{cc}
Z_{2}^{T} T_{1} T_{1}^{*} Z_{2} & Z_{2}^{T} T_{1} T_{2}^{*} Z_{1} \\
Z_{1}^{T} T_{2} T_{1}^{*} Z_{2} & Z_{1}^{T} T_{2} T_{2}^{*} Z_{1}
\end{array}\right] \geq 0
$$

if and only if

$$
Z_{1}^{T} T_{2} T_{2}^{*} Z_{1} \geq\left(Z_{1}^{T} T_{2} T_{1}^{*} Z_{2}\right)\left(Z_{2}^{T} T_{1} T_{1}^{*} Z_{2}\right)^{+}\left(Z_{2}^{T} T_{1} T_{2}^{*} Z_{1}\right)
$$


14 Generalization inequalities for Khatri-Rao product

Therefore (4.4) can be considered to be more general than (3.2). In order to prove this we set $T_{1}=I$ and $T_{2}=L$ in (4.4), we have

$$
\begin{aligned}
Z_{1}^{T} L L^{*} Z_{1} & \geq\left(Z_{1}^{T} L I^{*} Z_{2}\right)\left(Z_{2}^{T} I I^{*} Z_{2}\right)^{+}\left(Z_{2}^{T} I L^{*} Z_{1}\right) \\
& =\left(Z_{1}^{T} L Z_{2}\right)\left(Z_{2}^{T} Z_{2}\right)^{+}\left(Z_{2}^{T} L^{*} Z_{1}\right) \quad\left(Z_{2}^{T} Z_{2}=I\right) \\
& =\left(Z_{1}^{T} L Z_{2}\right)\left(Z_{1}^{T} L Z_{2}\right)^{*} .
\end{aligned}
$$

Returning to (4.4) and (3.2), it can be easily seen that various other choices of the matrices $T_{1}, T_{2}$, and $L$ are possible which lead to quite different inequalities involving Khatri-Rao products. However, there exist some inequalities that do not seem to follow directly from (1.7) or (2.11), but follow easily from (4.4) and (3.2). Based on (4.4) and (3.2) we generalize some inequalities in works of Visick [8, Corollary 13, Remark in page 56, Theorems 11, 17, and 20] and establish some new inequalities involving Khatri-Rao products of several positive matrices.

Theorem 4.1. Let $A_{1}$ and $A_{2}$ be compatible partitioned matrices. Then

$$
\begin{gathered}
A_{1} A_{1}^{*} * A_{2} A_{2}^{*}+A_{2} A_{2}^{*} * A_{1} A_{1}^{*}+A_{1} A_{2}^{*} * A_{2} A_{1}^{*}+A_{2} A_{1}^{*} * A_{1} A_{2}^{*} \\
\geq\left(A_{1} * A_{2}+A_{2} * A_{1}\right)\left(\left(A_{1} * A_{2}\right)^{*}+\left(A_{2} * A_{1}\right)^{*}\right) .
\end{gathered}
$$

Proof. Set $T_{1}=I \Theta I$ and $T_{2}=A_{1} \Theta A_{2}+A_{2} \Theta A_{1}$. Then calculations show that

$$
\begin{array}{r}
T_{2} T_{2}^{*}=A_{1} A_{1}^{*} \Theta A_{2} A_{2}^{*}+A_{2} A_{2}^{*} \Theta A_{1} A_{1}^{*}+A_{1} A_{2}^{*} \Theta A_{2} A_{1}^{*}+A_{2} A_{1}^{*} \Theta A_{1} A_{2}^{*}, \\
T_{2} T_{1}^{*}=A_{1} \Theta A_{2}+A_{2} \Theta A_{1}, \quad T_{1} T_{2}^{*}=\left(A_{1} \Theta A_{2}\right)^{*}+\left(A_{2} \Theta A_{1}\right)^{*}, \quad T_{1} T_{1}^{*}=I \Theta I .
\end{array}
$$

Substituting these into (4.4) and using (1.7), we get (4.6).

Corollary 4.2. Let $A_{i}(1 \leq i \leq 2)$ be Hermitian compatible partitioned matrices. Then

$$
A_{1}^{2} * A_{2}^{2} \geq\left(A_{1} * A_{2}\right)^{2}
$$

(ii)

$$
A^{2} * A^{-2} \geq\left(A * A^{-1}\right)^{2} \quad \text { if } A \text { is nonsingular; }
$$

(iii)

$$
I * A^{2} \geq(I * A)^{2} .
$$

Proof. (i) Set $A_{1}^{*}=A_{1}$ and $A_{2}^{*}=A_{2}$ in (3.14) of Corollary 3.3, we get (4.8).

(ii) Set $A_{1}=A$ and $A_{2}=A^{-1}$ in (4.8), we get (4.9).

(iii) Set $A_{1}=I$ and $A_{2}=A$ in (4.8), we get (4.10).

Corollary 4.3. Let $A_{i}>0(1 \leq i \leq 2)$ be compatible partitioned matrices. Then

$$
\left(A_{1}^{2} * A_{2}^{2}\right)^{1 / 2} \geq A_{1} * A_{2} \text {. }
$$


Proof. It follows immediately by (4.8) and Löwner-Heinz theorem.

Theorem 4.4. Let $A_{i} \geq 0(1 \leq i \leq k, k \geq 2)$ be compatible partitioned matrices and let $A_{i}^{0}=A_{i}^{1 / 2} A_{i}^{+1 / 2}=A_{i}^{+1 / 2} A_{i}^{1 / 2}(1 \leq i \leq k)$. Then

$$
\begin{aligned}
& 2\left(\prod_{i=1}^{k} * A_{i}^{0}\right)+\left(A_{1} * \prod_{i=2}^{k} * A_{i}^{+}\right)+\left(A_{1}^{+} * \prod_{i=2}^{k} * A_{i}\right) \\
& \quad \geq\left(A_{1} * \prod_{i=2}^{k} * A_{i}^{0}+A_{1}^{0} * \prod_{i=2}^{k} * A_{i}\right)\left(\prod_{i=1}^{k} * A_{i}\right)^{+}\left(A_{1} * \prod_{i=2}^{k} * A_{i}^{0}+A_{1}^{0} * \prod_{i=2}^{k} * A_{i}\right) .
\end{aligned}
$$

Proof. Since $A_{i} \geq 0(1 \leq i \leq k, k \geq 2)$, then $A_{i}^{*}=A_{i}$. Set $T_{1}=\prod_{i=1}^{k} \Theta A_{i}^{1 / 2}$ and $T_{2}=$ $A_{1}^{1 / 2} \Theta \prod_{i=2}^{k} \Theta A_{i}^{+1 / 2}+A_{1}^{+1 / 2} \Theta \prod_{i=2}^{k} A_{i}^{1 / 2}$. Since $A_{i}^{1 / 2} A_{i}^{1 / 2}=A_{i}, A_{i}^{+1 / 2} A_{i}^{+1 / 2}=A_{i}^{+}$, and $A_{i}^{0}=$ $A_{i}^{1 / 2} A_{i}^{+1 / 2}=A_{i}^{+1 / 2} A_{i}^{1 / 2}(1 \leq i \leq k)$, then calculations show that

$$
\begin{array}{r}
T_{2} T_{2}^{*}=2\left(\prod_{i=1}^{k} \Theta A_{i}^{0}\right)+\left(A_{1} \Theta \prod_{i=2}^{k} \Theta A_{i}^{+}\right)+\left(A_{1}^{+} \Theta \prod_{i=2}^{k} \Theta A_{i}\right), \quad T_{1} T_{1}^{*}=\prod_{i=1}^{k} \Theta A_{i}, \\
T_{2} T_{1}^{*}=\left(A_{1} \Theta \prod_{i=2}^{k} \Theta A_{i}^{0}+A_{1}^{0} \Theta \prod_{i=2}^{k} \Theta A_{i}\right), \quad T_{1} T_{2}^{*}=\left(A_{1} \Theta \prod_{i=2}^{k} \Theta A_{i}^{0}+A_{1}^{0} \Theta \prod_{i=2}^{k} \Theta A_{i}\right) .
\end{array}
$$

Substituting these into (4.4) and using Lemma 2.2, we get (4.12).

If we put $k=2$ and replace $A_{i}$ by $A_{i}^{r}(1 \leq i \leq 2)$ in Theorem 4.4 , we obtain the following theorem.

Theorem 4.5. Let $A_{1} \geq 0, A_{2} \geq 0$ be compatible partitioned and let $r$ be any nonzero real number such that $A_{1}^{0}=A_{1}^{r / 2} A_{1}^{+r / 2}=A_{1}^{+r / 2} A_{1}^{r / 2}$ and $A_{2}^{0}=A_{2}^{r / 2} A_{2}^{+r / 2}=A_{2}^{+r / 2} A_{2}^{r / 2}$. Then

$$
\begin{aligned}
& 2 A_{1}^{0} * A_{2}^{0}+A_{1}^{r} * A_{2}^{+r}+A_{1}^{+r} * A_{2}^{r} \\
& \quad \geq\left(A_{1}^{r} * A_{2}^{0}+A_{1}^{0} * A_{2}^{r}\right)\left(A_{1}^{r} * A_{2}^{r}\right)^{+}\left(A_{1}^{r} * A_{2}^{0}+A_{1}^{0} * A_{2}^{r}\right) .
\end{aligned}
$$

If $A_{1}>0, A_{2}>0$ in Theorem 4.5, we obtain the following theorem.

Theorem 4.6. Let $A_{1}>0, A_{2}>0$ be compatible partitioned and let I be a compatible partitioned identity matrix. Then for any nonzero real number $r$,

$$
2 I+A_{1}^{r} * A_{2}^{-r}+A_{1}^{-r} * A_{2}^{r} \geq\left(A_{1}^{r} * I+I * A_{2}^{r}\right)\left(A_{1}^{r} * A_{2}^{r}\right)^{-1}\left(A_{1}^{r} * I+I * A_{2}^{r}\right) .
$$

If we put $r=1$ and $A_{1}=A_{2}$ in Theorem 4.6, we obtain the following theorem.

Theorem 4.7. Let $A>0$ be compatible partitioned and let I be a compatible partitioned identity matrix. Then

$$
2 I+A * A^{-1}+A^{-1} * A \geq(A * I+I * A)(A * A)^{-1}(A * I+I * A) .
$$


16 Generalization inequalities for Khatri-Rao product

In particular, if I is a nonpartitioned identity matrix, then

$$
2 I+A * A^{-1}+A^{-1} * A \geq 4(I * A)(A * A)^{-1}(I * A) .
$$

Theorem 4.8. Let $A_{1}>0$ and $A_{2}>0$ be compatible partitioned matrices. Then for any nonzero real number $r$

$$
A_{1}^{r} * A_{2}^{-r}+A_{1}^{-r} * A_{2}^{r}+2 I \geq\left(A_{1}^{r / 2} * A_{2}^{-r / 2}+A_{1}^{-r / 2} * A_{2}^{r / 2}\right)^{2} .
$$

In particular, if $A_{1}=A_{2}=A$, Then

$$
A^{r} * A^{-r}+A^{-r} * A^{r}+2 I \geq\left(A^{r / 2} * A^{-r / 2}+A^{-r / 2} * A^{r / 2}\right)^{2} .
$$

Proof. Since $A_{1}>0$ and $A_{2}>0$, then $A_{1}^{*}=A_{1}$ and $A_{2}^{*}=A_{2}$. Set $L=A_{1}^{r / 2} \Theta A_{2}^{-r / 2}+$ $A_{1}^{-r / 2} \Theta A_{2}^{r / 2}$. Compute

$$
\begin{aligned}
Z_{1}^{T} L L^{*} Z_{1} & =Z_{1}^{T} L L Z_{1}=Z_{1}^{T}\left(A_{1}^{r / 2} \Theta A_{2}^{-r / 2}+A_{1}^{-r / 2} \Theta A_{2}^{r / 2}\right)\left(A_{1}^{r / 2} \Theta A_{2}^{-r / 2}+A_{1}^{-r / 2} \Theta A_{2}^{r / 2}\right) Z_{1} \\
& =Z_{1}^{T}\left(A_{1}^{r} \Theta A_{2}^{-r}\right) Z_{1}+Z_{1}^{T}(I \Theta I) Z_{1}+Z_{1}^{T}(I \Theta I) Z_{1}+Z_{1}^{T}\left(A_{1}^{-r} \Theta A_{2}^{r}\right) Z_{1} \\
& =A_{1}^{r} * A_{2}^{-r}+2 I+A_{1}^{-r} * A_{2}^{r} .
\end{aligned}
$$

Similarly,

$$
\begin{aligned}
\left(Z_{1}^{T} L Z_{2}\right)\left(Z_{1}^{T} L Z_{2}\right)^{*} & =\left(Z_{1}^{T} L Z_{2}\right)^{2}=\left\{Z_{1}^{T}\left(A_{1}^{r / 2} \Theta A_{2}^{-r / 2}+A_{1}^{-r / 2} \Theta A_{2}^{r / 2}\right) Z_{2}\right\}^{2} \\
& =\left(A_{1}^{r / 2} * A_{2}^{-r / 2}+A_{1}^{-r / 2} * A_{2}^{r / 2}\right)^{2}
\end{aligned}
$$

Substituting (4.20) and (4.21) into (3.2), we get (4.18).

From (4.18), we have the following special cases:

(i) for $r=1$, we have

$$
A_{1}^{*} A_{2}^{-1}+A_{1}^{-1} * A_{2}+2 I \geq\left(A_{1}^{1 / 2} * A_{2}^{-1 / 2}+A_{1}^{-1 / 2} * A_{2}^{1 / 2}\right)^{2}
$$

(ii) for $r=2$, we have

$$
A_{1}^{2} * A_{2}^{-2}+A_{1}^{-2} * A_{2}^{2}+2 I \geq\left(A_{1} * A_{2}^{-1}+A_{1}^{-1} * A_{2}\right)^{2} .
$$


From (4.19), we have the following special cases:

(i) for $r=1$, we have

$$
A * A^{-1}+A^{-1} * A+2 I \geq\left(A^{1 / 2} * A^{-1 / 2}+A^{-1 / 2} * A^{1 / 2}\right)^{2}
$$

(ii) for $r=2$, we have

$$
A^{2} * A^{-2}+A^{-2} * A^{2}+2 I \geq\left(A * A^{-1}+A^{-1} * A\right)^{2} .
$$

Theorem 4.9. Let $A_{1} \geq 0, A_{2} \geq 0$ be compatible partitioned and let I be a compatible partitioned identity matrix. Then

$$
A_{1}^{2} \infty A_{2}^{2}+2\left(A_{1} * A_{2}\right) \geq\left(A_{1} \infty A_{2}\right)^{2}
$$

where $A_{1} \infty A_{2}=A_{1} * I+I * A_{2}$ is called the Khatri-Rao sum.

Proof. Set $L=A_{1} \nabla A_{2}=A_{1} \Theta I+I \Theta A_{2}$ (Tracy-Singh sum). Since $A_{1} \geq 0$ and $A_{2} \geq 0$, then $A_{1}^{*}=A_{1}$ and $A_{2}^{*}=A_{2}$. Calculations show that

$$
\begin{aligned}
Z_{1}^{T} L L^{*} Z_{1} & =Z_{1}^{T} L L Z_{1}=Z_{1}^{T}\left(A_{1} \Theta I+I \Theta A_{2}\right)\left(A_{1} \Theta I+I_{2} \Theta A_{2}\right) Z_{1} \\
& =A_{1}^{2} * I+I * A_{2}^{2}+2\left(A_{1} * A_{2}\right)=A_{1}^{2} \infty A_{2}^{2}+2\left(A_{1} * A_{2}\right) .
\end{aligned}
$$

Similarly,

$$
\begin{aligned}
\left(Z_{1}^{T} L Z_{2}\right)\left(Z_{1}^{T} L Z_{2}\right)^{*} & =\left\{Z_{1}^{T}\left(A_{1} \Theta I+I \Theta A_{2}\right) Z_{2}\right\}\left\{Z_{1}^{T}\left(A_{1} \Theta I+I \Theta A_{2}\right) Z_{2}\right\}^{*} \\
& =\left(A_{1} * I+I * A_{2}\right)^{2}=\left(A_{1} \infty A_{2}\right)^{2} .
\end{aligned}
$$

Substituting (4.27) and (4.28) into (3.2), we get (4.26).

Theorem 4.10. Let $A_{1}>0$ and $A_{2}>0$ be compatible partitioned matrices. Then for any positive real number $r$,

$$
\begin{aligned}
& r\left(A_{1}^{2} * A_{2}^{2}\right)+\left(A_{1} A_{2} * A_{2} A_{1}\right)+\left(A_{2} A_{1} * A_{1} A_{2}\right)+\frac{1}{r}\left(A_{2}^{2} * A_{1}^{2}\right) \\
& \quad \geq r\left(A_{1} * A_{2}\right)^{2}+\left(A_{1} * A_{2}\right)\left(A_{2} * A_{1}\right)+\left(A_{2} * A_{1}\right)\left(A_{1} * A_{2}\right)+\frac{1}{r}\left(A_{2} * A_{1}\right)^{2} .
\end{aligned}
$$

Proof. Set $L=\varepsilon_{1} A_{1} \Theta A_{2}+\varepsilon_{2} A_{2} \Theta A_{1}$, where $\varepsilon_{1}$ and $\varepsilon_{2}$ are both positive. Since $A_{1}>0$ and $A_{2}>0$, then $A_{1}^{*}=A_{1}$ and $A_{2}^{*}=A_{2}$. Compute

$$
\begin{aligned}
Z_{1}^{T} L L^{*} Z_{1} & =Z_{1}^{T} L L Z_{1}=Z_{1}^{T}\left(\varepsilon_{1} A_{1} \Theta A_{2}+\varepsilon_{2} A_{2} \Theta A_{1}\right)\left(\varepsilon_{1} A_{1} \Theta A_{2}+\varepsilon_{2} A_{2} \Theta A_{1}\right) Z_{1} \\
& =Z_{1}^{T}\left\{\varepsilon_{1}^{2}\left(A_{1}^{2} \Theta A_{2}^{2}\right)+\varepsilon_{1} \varepsilon_{2}\left(A_{1} A_{2} \Theta A_{2} A_{1}\right)+\varepsilon_{1} \varepsilon_{2}\left(A_{2} A_{1} \Theta A_{1} A_{2}\right)+\varepsilon_{2}^{2}\left(A_{2}^{2} \Theta A_{1}^{2}\right)\right\} Z_{1} \\
& =\left\{\varepsilon_{1}^{2}\left(A_{1}^{2} * A_{2}^{2}\right)+\varepsilon_{1} \varepsilon_{2}\left(A_{1} A_{2} * A_{2} A_{1}\right)+\varepsilon_{1} \varepsilon_{2}\left(A_{2} A_{1} * A_{1} A_{2}\right)+\varepsilon_{2}^{2}\left(A_{2}^{2} * A_{1}^{2}\right)\right\} .
\end{aligned}
$$


Similarly,

$$
\begin{aligned}
\left(Z_{1}^{T} L Z_{2}\right)\left(Z_{1}^{T} L Z_{2}\right)^{*}= & \left\{Z_{1}^{T}\left(\varepsilon_{1} A_{1} \Theta A_{2}+\varepsilon_{2} A_{2} \Theta A_{1}\right) Z_{2}\right\}\left\{Z_{1}^{T}\left(\varepsilon_{1} A_{1} \Theta A_{2}+\varepsilon_{2} A_{2} \Theta A_{1}\right) Z_{2}\right\}^{*} \\
= & \left\{Z_{1}^{T}\left(\varepsilon_{1} A_{1} \Theta A_{2}+\varepsilon_{2} A_{2} \Theta A_{1}\right) Z_{2}\right\}\left\{Z_{1}^{T}\left(\varepsilon_{1} A_{1} \Theta A_{2}+\varepsilon_{2} A_{2} \Theta A_{1}\right) Z_{2}\right\} \\
= & \left\{\left(\varepsilon_{1} A_{1} * A_{2}+\varepsilon_{2} A_{2} * A_{1}\right)\right\}^{2} \\
= & \varepsilon_{1}^{2}\left(A_{1} * A_{2}\right)^{2}+\varepsilon_{1} \varepsilon_{2}\left(A_{1} * A_{2}\right)\left(A_{2} * A_{1}\right) \\
& +\varepsilon_{1} \varepsilon_{2}\left(A_{2} * A_{1}\right)\left(A_{1} * A_{2}\right)+\varepsilon_{2}^{2}\left(A_{2} * A_{1}\right)^{2} .
\end{aligned}
$$

Substituting (4.30) and (4.31) into (3.2), we have

$$
\begin{aligned}
& \left\{\varepsilon_{1}^{2}\left(A_{1}^{2} * A_{2}^{2}\right)+\varepsilon_{1} \varepsilon_{2}\left(A_{1} A_{2} * A_{2} A_{1}\right)+\varepsilon_{1} \varepsilon_{2}\left(A_{2} A_{1} * A_{1} A_{2}\right)+\varepsilon_{2}^{2}\left(A_{2}^{2} * A_{1}^{2}\right)\right\} \\
& \quad \geq\left\{\varepsilon_{1}^{2}\left(A_{1}^{*} A_{2}\right)^{2}+\varepsilon_{1} \varepsilon_{2}\left(A_{1} * A_{2}\right)\left(A_{2} * A_{1}\right)+\varepsilon_{1} \varepsilon_{2}\left(A_{2} * A_{1}\right)\left(A_{1} * A_{2}\right)+\varepsilon_{2}^{2}\left(A_{2}^{*} A_{1}\right)^{2}\right\} .
\end{aligned}
$$

Set $r=\varepsilon_{1} / \varepsilon_{2}$, we get (4.29).

Remark 4.11. Let $A_{i}(1 \leq i \leq k, k \geq 2)$ be compatible partitioned matrices. Then (3.7) can be proved by setting $T_{1}=\prod_{i=1}^{k} \Theta I$ and $T_{2}=\prod_{i=1}^{k} \Theta A_{i}$. Calculations show that

$$
T_{2} T_{2}^{*}=\prod_{i=1}^{k} \Theta A_{i} A_{i}^{*}, \quad T_{2} T_{1}^{*}=\prod_{i=1}^{k} \Theta A_{i}, \quad T_{1} T_{2}^{*}=\left(\prod_{i=1}^{k} \Theta A_{i}\right)^{*}, \quad T_{1} T_{1}^{*}=\prod_{i=1}^{k} \Theta I .
$$

Substituting these into (4.4) and using (2.11), we get (3.7).

Remark 4.12. Let $A_{i}(1 \leq i \leq 2)$ be compatible partitioned matrices. Then (3.14) can be proved by putting $k=2$ in Remark 4.11 .

Remark 4.13. All results obtained in Sections 3 and 4 are quite general. These results lead to inequalities involving Hadamard product, as a special case, for nonpartitioned matrices $A_{i}(i=1,2, \ldots, k, k \geq 2)$ with the Hadamard product and Kronecker product replacing the Khatri-Rao product and Tracy-Singh product, respectively.

Now we utilize the commutativity of the Hadamard product to develop, for instance, (3.7) of Theorem 3.2. This result leads to the following inequality involving Hadamard product, as a special case:

$$
\prod_{i=1}^{k} \circ\left(A_{i} A_{i}^{*}\right) \geq\left(\prod_{i=1}^{k} \circ\left(A_{i}\right)\right)\left(\prod_{i=1}^{k} \circ A_{i}\right)^{*} .
$$

It is possible to develop (4.34) in a different direction from (3.6). For example, Visick [8, Theorem 11, page 54] proved that if $A_{1}, A_{2} \in M_{m, n}$ and $s \in[-1,1]$, then

$$
A_{1} A_{1}^{*} \circ A_{2} A_{2}^{*}+s\left(A_{1} A_{2}^{*} \circ A_{2} A_{1}^{*}\right) \geq(1+s)\left(A_{1} \circ A_{2}\right)\left(A_{1} \circ A_{2}\right)^{*} .
$$


We will extend this inequality to the case of products involving any finite number of matrices.

If the Tracy-Singh and Khatri-Rao products are replaced by the Kronecker and Hadamard products in Lemma 2.2, respectively, we obtain the following corollary.

Corollary 4.14. Let $A_{i} \in M_{m, n}(1 \leq i \leq k, k \geq 2)$. Then

$$
\prod_{i=1}^{k} \circ A_{i}=P_{k m}^{T}\left(\prod_{i=1}^{k} \otimes A_{i}\right) P_{k n}
$$

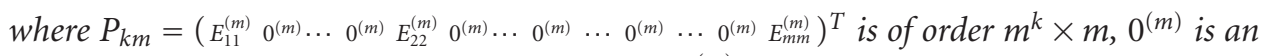
$m \times m$ matrix with all entries equal to zero, and $E_{i j}^{(m)}$ is an $m \times m$ matrix of zeros except for a one in the $(i, j)$ th position.

Theorem 4.15. Let $A_{i} \in M_{m, n}(1 \leq i \leq k, k \geq 2)$. Then for any real scalars $\alpha_{1}, \alpha_{2}, \ldots, \alpha_{k}$ which are not all zero,

$$
\begin{gathered}
\left(\alpha_{1}^{2}+\cdots+\alpha_{k}^{2}\right)\left(\prod_{i=1}^{k} \circ\left(A_{i} A_{i}^{*}\right)\right)+\left(\sum_{r=1}^{k-1} \mu_{r} \prod_{w=1}^{k} \circ\left(A_{w} A_{(w+r)^{\prime}}^{*}\right)\right) \\
\geq\left(\alpha_{1}+\cdots+\alpha_{k}\right)^{2}\left(\prod_{i=1}^{k} \circ A_{i}\right)\left(\prod_{i=1}^{k} \circ A_{i}\right)^{*},
\end{gathered}
$$

where $\mu_{r}=\sum_{w=1}^{k} \alpha_{w} \alpha_{(w+r)^{\prime}}$ and $w+r \equiv(w+r)^{\prime} \bmod k$ with $1 \leq(w+r)^{\prime} \leq k$.

Proof. Let

$L=\alpha_{1}\left(A_{1} \otimes A_{2} \otimes \cdots \otimes A_{k}\right)+\alpha_{2}\left(A_{2} \otimes \cdots \otimes A_{k} \otimes A_{1}\right)+\cdots+\alpha_{k}\left(A_{k} \otimes A_{1} \otimes \cdots \otimes A_{k-1}\right)$,

where $A_{i} \in M_{m, n}(1 \leq i \leq k, k \geq 2)$ and $\alpha_{1}, \alpha_{2}, \ldots, \alpha_{k}$ are real scalars which are not all zero. Taking indices “ $\bmod k$," Lemma 2.1(i), (iii) (by setting $\otimes$ instead of $\Theta$ ) give

$$
\begin{aligned}
L L^{*}= & \sum_{i=1}^{k} \alpha_{i}\left(A_{i} \otimes A_{i+1} \otimes \cdots \otimes A_{i-1}\right) \sum_{i=1}^{k} \alpha_{i}\left(A_{i}^{*} \otimes A_{i+1}^{*} \otimes \cdots \otimes A_{i-1}^{*}\right) \\
= & \alpha_{1}^{2}\left(A_{1} A_{1}^{*} \otimes \cdots \otimes A_{k} A_{k}^{*}\right)+\cdots+\alpha_{k}^{2}\left(A_{k} A_{k}^{*} \otimes A A_{1}^{*} \otimes \cdots \otimes A_{k} A_{k-1}^{*}\right) \\
& +\sum_{i \neq j} \alpha_{i} \alpha_{j}\left(A_{i} A_{j}^{*} \otimes A_{j+1} A_{j+1}^{*} \otimes \cdots \otimes A_{j-1} A_{j-1}^{*}\right) .
\end{aligned}
$$

Now the application of (4.36) and the commutativity of the Hadamard product yield

$$
P_{k m}^{T} L L^{*} P_{k m}=\left(\alpha_{1}^{2}+\cdots+\alpha_{k}^{2}\right)\left(\prod_{i=1}^{k} \circ\left(A_{i} A_{i}^{*}\right)\right)+\left(\sum_{r=1}^{k-1} \mu_{r} \prod_{w=1}^{k} \circ\left(A_{w} A_{(w+r)^{\prime}}^{*}\right)\right),
$$

where $\mu_{r}=\sum_{w}^{k} \alpha_{w} \alpha_{(w+r)^{\prime}}$ and $w+r \equiv(w+r)^{\prime} \bmod k$ with $1 \leq(w+r)^{\prime} \leq k$. 
Also by (4.36) and the commutativity of the Hadamard product, we obtain

$$
\begin{aligned}
\left(P_{k m}^{T} L P_{k n}\right)= & P_{k m}^{T}\left\{\alpha_{1}\left(A_{1} \otimes A_{2} \otimes \cdots \otimes A_{k}\right)+\alpha_{2}\left(A_{2} \otimes \cdots \otimes A_{k} \otimes A_{1}\right)\right. \\
& \left.+\cdots+\alpha_{k}\left(A_{k} \otimes A_{1} \otimes \cdots \otimes A_{k-1}\right)\right\} P_{k n} \\
= & \alpha_{1} P_{k m}^{T}\left(A_{1} \otimes A_{2} \otimes \cdots \otimes A_{k}\right) P_{k n}+\alpha_{2} P_{k m}^{T}\left(A_{2} \otimes \cdots \otimes A_{k} \otimes A_{1}\right) P_{k n} \\
& +\cdots+\alpha_{k} P_{k m}^{T}\left(A_{k} \otimes A_{1} \otimes \cdots \otimes A_{k-1}\right) P_{k n} \\
= & \alpha_{1}\left(A_{1} \circ A_{2} \circ \cdots \circ A_{k}\right)+\alpha_{2}\left(A_{2} \circ \cdots \circ A_{k} \circ A_{1}\right) \\
& +\cdots+\alpha_{k}\left(A_{k} \circ A_{1} \circ \cdots \circ A_{k-1}\right) \\
& \left(\alpha_{1}+\cdots+\alpha_{k}\right)\left(\prod_{i=1}^{k} \circ A_{i}\right) \\
\left(P_{k m}^{T} L P_{k n}\right)^{*}= & \left(\alpha_{1}+\cdots+\alpha_{k}\right)\left(\prod_{i=1}^{k} \circ A_{i}\right)
\end{aligned}
$$

Now

$$
\left(P_{k m}^{T} L P_{k n}\right)\left(P_{k m}^{T} L P_{k n}\right)^{*}=\left(\alpha_{1}+\cdots+\alpha_{k}\right)^{2}\left(\prod_{i=1}^{k} \circ A_{i}\right)\left(\prod_{i=1}^{k} \circ A_{i}\right)^{*} .
$$

Since $P_{k m}^{T} L L^{*} P_{k m} \geq\left(P_{k m}^{T} L P_{k n}\right)\left(P_{k m}^{T} L P_{k n}\right)^{*}$ by (3.2) and from (4.40) and (4.42), we get (4.37).

Now, we examine some special cases briefly.

In order to see that (4.37) really is an extension in (4.34), it is sufficient to set $\alpha_{1}=1$ and $\alpha_{2}=\cdots=\alpha_{k}=0$. Thus we recover the result of Visick in (4.35) which we mentioned before the statement of Corollary 4.14. Let $k=2$, then $\mu_{1}=\sum_{w=1}^{2} \alpha_{w} \alpha_{(w+1)^{\prime}}$ with $w+1 \equiv$ $(w+1)^{\prime} \bmod 2$, that is, $\mu_{1}=2 \alpha_{1} \alpha_{2}$. Then Theorem 4.15 asserts that

$$
\left(\alpha_{1}^{2}+\alpha_{2}^{2}\right)\left(A_{1} A_{1}^{*} \circ A_{2} A_{2}^{*}\right)+2 \alpha_{1} \alpha_{2}\left(A_{1} A_{2}^{*} \circ A_{2} A_{1}^{*}\right) \geq\left(\alpha_{1}+\alpha_{2}\right)^{2}\left(A_{1} \circ A_{2}\right)\left(A_{1} \circ A_{2}\right)^{*} \text {. }
$$

Simplification gives

$$
A_{1} A_{1}^{*} \circ A_{2} A_{2}^{*}+s\left(A_{1} A_{2}^{*} \circ A_{2} A_{1}^{*}\right) \geq(1+s)\left(A_{1} \circ A_{2}\right)\left(A_{1} \circ A_{2}\right)^{*}
$$

for any $s \in[-1,1]$, just as we wanted. Finally, we present an attractive inequality using three matrices. Let $k=3, \alpha_{1}=1, \alpha_{2}=\alpha_{3}=-1 / 2$. Theorem 4.15 asserts that

$$
A_{1} A_{1}^{*} \circ A_{2} A_{2}^{*} \circ A_{3} A_{3}^{*} \geq \frac{1}{2}\left\{A_{1} A_{2}^{*} \circ A_{2} A_{3}^{*} \circ A_{3} A_{1}^{*}+A_{2} A_{1}^{*} \circ A_{3} A_{2}^{*} \circ A_{1} A_{3}^{*}\right\} .
$$




\section{Acknowledgments}

The authors would like to thank the referees for their valuable comments and suggestions, including the simplified proof of Theorem 3.6 and some statements. The present research has been partially supported by University Putra Malaysia (UPM) under the Grant IRPA09-02-04-0259-EA001.

\section{References}

[1] Z. A. Al Zhour and A. Kilicman, New Holder-type inequalities for the Tracy-Singh and KhatriRao products of positive matrices, Proceedings of the International Conference on Mathematics, Statistics and Their Applications, vol. 1, North Sumatera, 2005, pp. 1-7.

[2] A. Albert, Conditions for positive and nonnegative definiteness in terms of pseudoinverses, SIAM Journal on Applied Mathematics 17 (1969), no. 2, 434-440.

[3] C.-G. Cao, X. Zhang, and Z.-P. Yang, Some inequalities for the Khatri-Rao product of matrices, Electronic Journal of Linear Algebra 9 (2002), 276-281.

[4] J. Chollet, Some inequalities for principal submatrices, The American Mathematical Monthly 104 (1997), no. 7, 609-617.

[5] S. Liu, Matrix results on the Khatri-Rao and Tracy-Singh products, Linear Algebra and Its Applications 289 (1999), no. 1-3, 267-277.

[6] _ Several inequalities involving Khatri-Rao products of positive semidefinite matrices, Linear Algebra and Its Applications 354 (2002), no. 1-3, 175-186.

[7] B. Mond and J. E. Pečarić, Matrix inequalities for convex functions, Journal of Mathematical Analysis and Applications 209 (1997), no. 1, 147-153.

[8] G. Visick, A quantitative version of the observation that the Hadamard product is a principal submatrix of the Kronecker product, Linear Algebra and Its Applications 304 (2000), no. 1-3, 45-68.

[9] F. Zhang, Matrix Theory. Basic Results and Techniques, Universitext, Springer, New York, 1999.

[10] X. Zhang, Z.-P. Yang, and C.-G. Cao, Inequalities involving Khatri-Rao products of positive semidefinite matrices, Applied Mathematics E-Notes 2 (2002), 117-124.

Zeyad Abdel Aziz Al Zhour: Department of Mathematics and Institute for Mathematical Research, University Putra Malaysia (UPM), 43400 Serdang, Selangor, Malaysia

E-mail address: zeyad1968@yahoo.com

Adem Kilicman: Department of Mathematics and Institute for Mathematical Research, University Putra Malaysia (UPM), 43400 Serdang, Selangor, Malaysia

E-mail address: akilic@fsas.upm.edu.my 